\title{
Genetic variant of PRKAA1 and gastric cancer risk in an Eastern Chinese population
}

\author{
Li-Xin Qiu' ${ }^{1,2, *}$, Jing $\mathrm{He}^{3, *}$, Lei Cheng ${ }^{2, *}$, Fei Zhou ${ }^{2}$, Meng-Yun Wang ${ }^{2}$, Meng-Hong \\ Sun ${ }^{4}$, Xiao-Yan Zhou ${ }^{4}$, Jin Li $^{1}$, Wei-Jian Guo ${ }^{1}$, Ya-Nong Wang ${ }^{5}$, Ya-Jun Yang ${ }^{6,7}$, \\ Jiu-Cun Wang ${ }^{6,7}$, Li Jin' ${ }^{6,7}$, Xiao-Dong Zhu' ${ }^{1}$, Qing-Yi Wei ${ }^{2,8}$ \\ ${ }^{1}$ Department of Medical Oncology, Fudan University Shanghai Cancer Center, Department of Oncology, Shanghai Medical \\ College, Fudan University, Shanghai, China \\ ${ }^{2}$ Cancer Institute, Collaborative Innovation Center for Cancer Medicine, Fudan University Shanghai Cancer Center, Shanghai, \\ China \\ ${ }^{3}$ Department of Pediatric Surgery, Guangzhou Women and Children's Medical Center, Guangzhou Medical University, \\ Guangzhou, China \\ ${ }^{4}$ Department of Pathology, Fudan University Shanghai Cancer Center, Shanghai, China \\ ${ }^{5}$ Department of Gastric Cancer and Soft Tissue Sarcoma Surgery, Fudan University Shanghai Cancer Center, Shanghai, China \\ ${ }^{6}$ Ministry of Education Key Laboratory of Contemporary Anthropology and State Key Laboratory of Genetic Engineering, \\ School of Life Sciences, Fudan University, Shanghai, China \\ ${ }^{7}$ Fudan-Taizhou Institute of Health Sciences, Jiangsu, China \\ ${ }^{8}$ Duke Cancer Institute, Duke University Medical Center, Durham, NC, USA \\ *These authors have contributed equally to this work
}

Correspondence to: Xiao-Dong Zhu, e-mail: xddr@netease.com

Qing-Yi Wei, e-mail: weiqingyi@yahoo.com and Qingyi.wei@duke.edu

Keywords: PRKA71, polymorphism, gastric cancer, genetic susceptibility

Received: August 04, 2015

Accepted: October 09, 2015

Published: October 15, 2015

\section{ABSTRACT}

Published data on the association between PRKAA1 rs13361707 T > C polymorphism and gastric cancer (GCa) susceptibility were inconclusive. To derive a more precise estimation of the association, we conducted a large-scale GCa study of 1,124 cases and 1,194 controls to confirm this association in an Eastern Chinese population. Our results showed that the C allele of PRKAA1 rs13361707 increased the GC risk in the study population [CT vs. TT, odds ratio $(O R)=1.72,95 \%$ confidence interval $(\mathrm{CI})=1.40-2.12 ; \mathrm{CC}$ vs. TT, OR $=2.15,95 \% \mathrm{CI}=1.70-2.71 ; \mathrm{CT} / \mathrm{CC}$ vs. TT, $\mathrm{OR}=1.86,95 \% \mathrm{CI}=1.53-2.26 ; \mathrm{CC}$ vs.TT $/ \mathrm{CT}, \mathrm{OR}=1.49,95 \% \mathrm{CI}=1.24-1.79]$. In addition, the association of $\mathrm{C}$ allele with an increased $\mathrm{GCa}$ risk was still significant in subgroups, when stratified by age, sex, tumor site, drinking and smoking status. Moreover, the findings in the present study were validated by our further meta-analysis. In summary, these results indicated that the C allele of PRKAA1 rs13361707 was a low-penetrate risk factor for GCa.

\section{INTRODUCTION}

Gastric cancer (GCa) is currently the most frequently occurring cancer and one of the leading causes of cancer-related death in the world. A total of 951,600 new GCa cases and 723,100 deaths are estimated to have occurred in 2012, accounting for $8 \%$ of the total cases and $10 \%$ of total deaths [1]. Although mechanism of gastric carcinogenesis is still not fully understood, it has been suggested that environmental factors combining with low-penetrance susceptibility genes may be important. For example, a high rate of Helicobacter pylori (HP) infection (70-90\%) in developing countries (compared with $25-50 \%$ in developed countries) might be a potential risk factor for $\mathrm{GCa}[2,3]$. However, because only few $H P$ carriers eventually develop $\mathrm{GCa}$, other factors must play a role in GCa risk. Life styles, such as tobacco smoking and diet, are also suggested as potential risk factors for 
GCa [4], but the relevant data are limited. On the other hand, although studies have shown an association between high body mass index and GCa risk in developed countries [5], this association is weak in Chinese populations [6]. Up to now, genetic factors for GCa risk are still not fully recognized.

Recent studies have shown significant associations between genetic variants and GCa risk [7-10], but additional confirmation in different population is needed. For example, three GCa GWAS studies reported that SNPs in PSCA, MUC1, and PLCE1 were associated with an increased GCa risk [11-13]. More recently, the rs13361707 SNP in PRKAA1 (encoding protein kinase, AMP-activated, alpha 1 catalytic subunit) pathway was newly identified by a GWAS study in Chinese Han population as a risk factor for non-cardia $\mathrm{GCa}$ [14].

The rs13361707 SNP is located in the first intron of PRKAA1 at 5p13.1. The PRKAA1 protein is one of the subunits of the mammalian 5'-AMP-activated protein kinase (AMPK), a central metabolic switch found in all eukaryotes that governs glucose and lipid metabolism in response to alterations in nutrient and intracellular energy levels [15]. AMPK has been implicated in a number of diseases related to energy metabolism, including cancer.

However, it was noted that Chinese populations included in these GWAS studies and replications were mostly northern (e.g., Beijing City) and southern Chinese (e.g., Nanjing City) populations, and the results in these reports were not always consistent. Of note, one case-control study did not support the association between rs13361707 SNP and GCa risk [16]. Moreover, some bias was considered inevitable in the published studies because of population stratification.

To fill these gaps, first, we conducted a replication study on the association between PRKAA1 rs13361707 SNP and GCa risk in a large eastern Chinese population rarely included in previous studies. Second, to avoid regional bias, which may be caused by different genetic background, we carried out a meta-analysis to increase statistical power for assessing the association between PRKAA1 rs13361707 SNP and GCa risk.

\section{RESULTS}

Baseline information of the study population was similar to our previous study [17]. One sample in cases and two samples in controls failed to be genotyped. Thus, a total of 1,124 GCa patients and 1,194 cancerfree controls were included in the final analysis. Individuals were well matched by age and sex. The allele frequency of this SNP in control group was in line with HWE.

Table 1 listed the allele frequency of rs13361707 $\mathrm{T}>\mathrm{C}$ SNP in cases and controls and the association of the rs13361707 $\mathrm{T}>\mathrm{C}$ SNP with GCa risk. The results indicated that the $\mathrm{C}$ allele of PRKAAl rs13361707 increases $\mathrm{GC}$ risk[CT vs. TT, odds ratio $(\mathrm{OR})=1.72$, $95 \%$ confidence interval $(\mathrm{CI})=1.40-2.12$; CC vs. TT, OR $=2.15,95 \% \mathrm{CI}=1.70-2.71 ; \mathrm{CT} / \mathrm{CC}$ vs. TT, $\mathrm{OR}=1.86$, $95 \% \mathrm{CI}=1.53-2.26 ; \mathrm{CC}$ vs.TT/CT, OR $=1.49,95 \% \mathrm{CI}=$ $1.24-1.79$; and additive model, $\mathrm{OR}=1.46,95 \% \mathrm{CI}=1.30$ $1.64]$ in the study population. The population attributable risk of $\mathrm{CT}, \mathrm{CC}, \mathrm{CT} / \mathrm{CC}$ alleles were $0.53 \%, 11.51 \%$ and $4.4 \%$ respectively.

In the stratified analysis presented in Table 2, no significant heterogeneity was found between subgroups stratified by different factors. The association between rs13361707 SNP and GCa risk was not altered by age, sex, drinking status, smoking status, and tumor site. Using 0.0038 as the significance level of Bonferroni correction for multiple testing, most results were still statistically significant.

For the subsequent meta-analysis, we found four primary studies [16, 24-26]. By including the present study, a total of five studies with 9,590 cases and 9,724 controls were included in the meta-analysis. Pooled data indicated that the PRKAAlrs13361707 T > C SNP was strongly associated with increased $\mathrm{GCa}$ risk in several genetic model (CT vs. TT: $\mathrm{OR}=1.35,95 \% \mathrm{CI}=1.12-1.61$; $\mathrm{CC}$ vs. TT: $\mathrm{OR}=1.70,95 \% \mathrm{CI}=1.34-2.16$; and $\mathrm{CT} / \mathrm{CC}$ vs. TT: $\mathrm{OR}=1.44,95 \% \mathrm{CI}=1.17-1.76$, Supplementary Figure 1) without significant publication bias. Because of the notable heterogeneity across the studies, the leave-one-out sensitivity analysis was performed, and the study reported by Dong et al. [16] was considered the source of heterogeneity (dominant: $\mathrm{I}^{2}, 83.1 \%-4.8 \%$; homozygous: $\mathrm{I}^{2}, 82.3 \%-0.1 \%$; and heterozygous: $\mathrm{I}^{2}, 76.1 \%-0.0 \%$ ). More importantly, the pooled data from the final four studies still showed a strong association between rs13361707 and GCa risk, indicating the stability of the results of this meta-analysis.

\section{DISCUSSION}

Apart from environmental and lifestyle factors for GCa risk, genetic factors in the GCa development were important in identifying at-risk populations for cancer prevention. To our knowledge, the present study was the first to demonstrate the strong association of rs13361707 SNP with an increased GCa risk in a large eastern Chinese population under five genetic models. All results consistently showed that the $\mathrm{C}$ allele of rs 13361707 increased the risk of GCa.

PRKAA1, a subunit of the AMPK pathway, is critical to cellular activity and cancer development, and studies have demonstrated its role in cell differentiation, apoptosis, autophagy, and cancer progression [27-30] as well as in clinical prognosis $[31,32]$. Recently, targeting PRKKA1 was reported as a potential way for cancer suppression [30]. Although lacking of data about the biological mechanism of the PRKKA1 pathway in GCa, the corresponding rs13361707 SNP was postulated to active the expression of PRKKA1. 
Table 1: Logistic Regression Analysis of Associations between the Genotypes of PRKAA1 rs13361707 $\mathbf{T}>\mathrm{C}$ and Gastric Cancer Risk in an Eastern Chinese Population

\begin{tabular}{|c|c|c|c|c|c|c|}
\hline Genotype & $\begin{array}{c}\text { Cases } \\
(N=1,124)\end{array}$ & $\begin{array}{l}\text { Controls } \\
(N=1,194)\end{array}$ & $\begin{array}{c}\text { Crude OR } \\
(95 \% \text { CI })\end{array}$ & $P$ & $\begin{array}{l}\text { Adjusted OR } \\
(95 \% \mathrm{CI})^{\mathrm{a}}\end{array}$ & $P^{\text {a }}$ \\
\hline TT & $209(18.6)$ & $356(29.8)$ & 1.00 & & 1.00 & \\
\hline $\mathrm{CT}$ & $571(50.8)$ & $565(47.3)$ & $1.72(1.40-2.12)$ & $3.7 * 10^{-7}$ & $1.74(1.42-2.14)$ & $1.6 * 10^{-7}$ \\
\hline $\mathrm{CC}$ & $344(30.6)$ & $273(22.9)$ & $2.15(1.70-2.71)$ & $9.1 * 10^{-11}$ & $2.18(1.73-2.76)$ & $9.5 * 10^{-11}$ \\
\hline $\mathrm{CT} / \mathrm{CC}$ & $915(81.4)$ & $838(81.2)$ & $1.86(1.53-2.26)$ & $4.3 * 10^{-10}$ & $1.89(1.55-2.29)$ & $4.3 * 10^{-10}$ \\
\hline Additive & & & $1.46(1.30-1.64)$ & $1.8^{*} 10^{-10}$ & $1.47(1.31-1.65)$ & $6.3 * 10^{-11}$ \\
\hline $\mathrm{TT} / \mathrm{CT}$ & $780(69.4)$ & $921(77.1)$ & 1.00 & & 1.00 & \\
\hline $\mathrm{CC}$ & $344(30.6)$ & $273(22.9)$ & $1.49(1.24-1.79)$ & $2.0 * 10^{-5}$ & $1.50(1.25-1.81)$ & $2.3 * 10^{-5}$ \\
\hline
\end{tabular}

CI, confidence interval; OR, odds ratio

${ }^{a}$ Adjusted for age, sex, smoking and drinking status in logistic regression models

Table 2: Stratification analysis for the association between PRKAA1 rs13361707 T > C polymorphism and GC risk

\begin{tabular}{|c|c|c|c|c|c|c|c|}
\hline Variables & $\begin{array}{c}\text { rs13361707 } \\
\text { (cases/ } \\
\text { controls) }\end{array}$ & $\mathrm{CT} / \mathrm{CC}$ & $\begin{array}{c}\text { Crude OR } \\
95 \% \text { CI }\end{array}$ & $P$ & $P^{\text {het }}$ & $\begin{array}{l}\text { Adjusted OR }{ }^{\mathrm{a}} \\
\mathbf{9 5 \%} \mathrm{CI}\end{array}$ & $P^{a^{a}}$ \\
\hline \multicolumn{8}{|c|}{ Median age, yr } \\
\hline$\leq 59$ & $110 / 166$ & $468 / 440$ & $1.61(1.22-2.11)$ & $5.6^{*} 10^{-4}$ & $1.2 * 10^{-1}$ & $1.61(1.22-2.12)$ & $6.9 * 10^{-4}$ \\
\hline$>59$ & $99 / 190$ & $447 / 398$ & $2.16(1.63-2.85)$ & $5.1 * 10^{-8}$ & & $2.20(1.66-2.91)$ & $3.3 * 10^{-8}$ \\
\hline \multicolumn{8}{|l|}{ Sex } \\
\hline Males & $151 / 243$ & $649 / 584$ & $1.79(1.42-2.26)$ & $9.9 * 10^{-7}$ & $5.9 * 10^{-1}$ & $1.82(1.44-2.30)$ & $5.3 * 10^{-7}$ \\
\hline Females & $58 / 113$ & $266 / 254$ & $2.04(1.42-2.93)$ & $1.1 * 10^{-4}$ & & $2.05(1.42-2.94)$ & $9.5 * 10^{-5}$ \\
\hline \multicolumn{8}{|c|}{ Smoking status } \\
\hline Never & $127 / 192$ & $558 / 416$ & $2.03(1.57-2.62)$ & $5.4^{*} 10^{-8}$ & $3.4 * 10^{-1}$ & $2.05(1.58-2.66)$ & $6.6^{*} 10^{-8}$ \\
\hline Ever & $82 / 164$ & $357 / 422$ & $1.69(1.25-2.28)$ & $6.0^{*} 10^{-4}$ & & $1.69(1.25-2.28)$ & $5.9 * 10^{-4}$ \\
\hline \multicolumn{8}{|l|}{ Pack-year } \\
\hline 0 & $127 / 192$ & $558 / 416$ & $2.03(1.57-2.62)$ & $5.4^{*} 10^{-8}$ & $4.0^{*} 10^{-1}$ & $2.05(1.58-2.66)$ & $6.6 * 10^{-8}$ \\
\hline$\leq 25$ (mean) & $43 / 110$ & $184 / 243$ & $1.94(1.30-2.89)$ & $1.2 * 10^{-3}$ & & $1.87(1.24-2.81)$ & $3.0^{*} 10^{-3}$ \\
\hline$>25$ (mean) & $39 / 54$ & $173 / 179$ & $1.34(0.84-2.12)$ & $2.2 * 10^{-1}$ & & $1.40(0.86-2.28)$ & $1.8^{*} 10^{-1}$ \\
\hline \multicolumn{8}{|c|}{ Drinking status } \\
\hline Never & $161 / 260$ & $693 / 589$ & $1.90(1.52-2.38)$ & $2.3 * 10^{-8}$ & $7.3 * 10^{-1}$ & $1.93(1.54-2.42)$ & $1.2 * 10^{-8}$ \\
\hline Ever & $48 / 96$ & $222 / 249$ & $1.78(1.21-2.64)$ & $4.1 * 10^{-3}$ & & $1.78(1.21-2.64)$ & $4.1 * 10^{-3}$ \\
\hline \multicolumn{8}{|l|}{ Tumor site } \\
\hline GCA & $65 / 356$ & $240 / 838$ & $1.57(1.16-2.12)$ & $3.2 * 10^{-3}$ & $2.2 * 10^{-1}$ & $1.60(1.18-2.17)$ & $2.5^{*} 10^{-3}$ \\
\hline NGCA & $144 / 356$ & $675 / 838$ & $1.99(1.60-2.48)$ & $8.9 * 10^{-10}$ & & $2.02(1.62-2.51)$ & $2.2 * 10^{-10}$ \\
\hline
\end{tabular}

${ }^{a}$ Adjusted for age, sex, smoking and drinking status in logistic regression models 
The major finding in the present study is a confirmed strong association between PRKAA1rs13361707 in GCa risk in an eastern Chinese population under several genetic models. However, a previous GWAS study [14] confined a significant association between rs13361707 and GCa risk to the subsets of non-cardia GCa. Another replication study in Korea populations [26] indicated that rs13361707 is a risk factor for both GCA and NGCA, a consistent finding with the present study. The inconsistence of our results with the previously published GWAS study might be due to low statistical power in the analysis of subgroups stratified by tumor site. On the other hand, despite of a strong association of rs13361707 $\mathrm{T}>\mathrm{C}$ polymorphism with cardia GCa or no-cardia GCa in several studies, the present study indicated a similar trend in comparison with the GWAS study by Shi et al [13] that the rs13361707 SNP was more strongly associated with tumors in non-cardia site than tumors in cardia site. However, these results should be verified by future studies with a larger sample size.

There are some limitations in the present study. First, although age, sex, smoking status, drinking status, and tumor site were taken into consideration for subgroup analysis, other important risk factors, such as diet and HP infection, that were missing in this study, which may have also contributed to the etiology of GCa. New classification of tumor types is also important, which may have a different genetic basis in the etiology [33]. Second, the sample size of the cases in each of subgroups was largely reduced in the stratification analysis, which may have led to limited statistical power in subsequent analysis.

In conclusion, the present study confirmed that the $\mathrm{C}$ allele of PRKAA1 rs13361707 was a risk factor for GCa. However, future studies should incorporate $H P$ infection status and Lauren classification, which may lead to our better, comprehensive understanding of the association between the PRKAA1 rs13361707 SNP and GCa risk.

\section{MATERIALS AND METHODS}

\section{Study subjects}

This study included patients who were recruited from our ongoing molecular epidemiology study of GCa, and the cases and controls were described previously [17-19]. Briefly, 1,125 unrelated ethnic Han Chinese patients with newly diagnosed and histopathologically confirmed primary GCa were recruited from Fudan University Shanghai Cancer Center (FUSCC) in Eastern China between January 2009 and March 2011. Patients other than histopathologically confirmed primary GCa were excluded. In addition, 1,196 age and sex-matched cancer-free ethnic Han Chinese controls were recruited from the Taizhou Longitudinal (TZL) study conducted at the same time period in Eastern China as described previously [20]. Blood samples of GCa patients and cancer-free controls were provided by the tissue bank of FUSCC and the TZL study, respectively. All subjects had signed a written informed consent for donating their biological samples to the tissue bank for scientific research. Demographic data and environmental exposure history of each patient were collected. The overall response rate was approximately $91 \%$ for cases and $90 \%$ for controls. This research protocol was approved by the FUSCC institutional review board.

\section{SNP genotyping}

According to a relevant protocol, we extracted DNA from peripheral blood. The rs13361707 SNP was genotyped by the TaqMan assay with ABI7900HT real-time PCR system as reported previously [17]. Patients' status was unrevealed in the genotyping process. As recommend by the company, four negative controls (without DNA template) and two duplicated samples were included in each 384-plate for the quality control. The assays were repeated for $5 \%$ of the samples, and the results were $100 \%$ concordant.

\section{Statistical methods}

The $\chi^{2}$ test was used to assess the differences in the distribution of demographic characteristics between cases and controls. Hardy-Weinberg equilibrium (HWE) was tested by goodness-of-fit $\chi^{2}$ to evaluate the expected distributions of genetic frequency in the controls. The association between SNP and GCa risk was assessed by odds ratio (OR) and 95\% confidence intervals (CIs) in heterozygous (CT vs TT), homozygous (CC vs TT), dominant $(\mathrm{CT}+\mathrm{CC}$ vs TT), recessive (CC vs $\mathrm{CT}+\mathrm{TT})$, respectively. $\mathrm{OR}$ values were calculated by univariate and multivariate logistic regression models. Moreover, logistic regression tests for each genetic model were adjusted for age, sex, drinking and smoking status. Furthermore, associations between the PRKAA1 rs13361707 SNP and GCa risk were also stratified by age, sex, smoking or drinking status, and primary tumor site. We applied Bonferroni correction for multiple testing using 0.0038 as significance level. All statistical process above was achieved by SAS software (version 9.1; SAS Institute, Cary, NC)

To validate our results, we performed a mini meta-analysis with studies searched from Medline, PubMed and Embase. After using the search terms and inclusion and exclusion criteria as described in previous studies $[21,22]$, all primary reports were carefully reviewed, and the relevant references in these papers were also searched and reviewed by two independent authors. Then, data were retrieved from the reported studies and pooled crude ORs for heterozygous, homozygous, and dominant models were calculated. Heterogeneity between studies was estimated by Chi-square-based Q test. Pooled Ors were calculated by a fixed-effects model or random-effects model, depending on the heterogeneity between searched 
studies [23]. To validate the stability of the pooled results and to identify the sources of heterogeneity, the leave-oneout sensitive analysis was performed. Publication bias was shown by the funnel plot, in which the asymmetry will be estimated by Egger's liner regression test, where the statistically significant publication bias was tested out, when $P<0.05$ determined by the $t$ test as suggested by Egger. All statistical process was achieved by STATA version 10.0 (Stata Corporation, College Station, TX).

\section{ACKNOWLEDGMENTS AND FUNDINGS}

This study was supported by grant from the National Natural Science Foundation of China (81101808), the grant from "China's Thousand Talents Program" Recruitment at Fudan University, and the grant from the Ministry of Health (201002007).

\section{CONFLICTS OF INTEREST}

None declared.

\section{REFERENCES}

1. Torre LA, Bray F, Siegel RL, Ferlay J, Lortet-Tieulent J, Jemal A. Global cancer statistics, 2012. CA Cancer J Clin. 2015; 65:87-108.

2. Ye W, Held M, Lagergren J, Engstrand L, Blot WJ, McLaughlin JK, Nyren O. Helicobacter pylori infection and gastric atrophy: risk of adenocarcinoma and squamouscell carcinoma of the esophagus and adenocarcinoma of the gastric cardia. J Natl Cancer Inst. 2004; 96:388-396.

3. Ishaq S, Nunn L. Helicobacter pylori and gastric cancer: a state of the art review. Gastroenterol Hepatol Bed Bench. 2015; 8:S6-S14.

4. Woo HD, Lee J, Choi IJ, Kim CG, Lee JY, Kwon O, Kim J. Dietary flavonoids and gastric cancer risk in a Korean population. Nutrients. 2014; 6:4961-4973.

5. Engel LS, Chow WH, Vaughan TL, Gammon MD, Risch HA, Stanford JL, Schoenberg JB, Mayne ST, Dubrow R, Rotterdam H, West AB, Blaser M, Blot WJ, Gail MH, Fraumeni JF, Jr. Population attributable risks of esophageal and gastric cancers. J Natl Cancer Inst. 2003; 95: 1404-1413.

6. Tran GD, Sun XD, Abnet CC, Fan JH, Dawsey SM, Dong ZW, Mark SD, Qiao YL, Taylor PR. Prospective study of risk factors for esophageal and gastric cancers in the Linxian general population trial cohort in China. Int $\mathrm{J}$ Cancer. 2005; 113:456-463.

7. Zhang W, Liu H, Liu Z, Zhu D, Amos CI, Fang S, Lee JE, Wei Q. Functional Variants in Notch Pathway Genes NCOR2, NCSTN, and MAML2 Predict Survival of Patients with Cutaneous Melanoma. Cancer Epidemiol Biomarkers Prev. 2015; 24:1101-1110.
8. Shi TY, He J, Wang MY, Zhu ML, Yu KD, Shao ZM, Sun MH, Wu X, Cheng X, Wei Q. CASP7 variants modify susceptibility to cervical cancer in Chinese women. Sci Rep. 2015; 5:9225.

9. Zhao Y, Wei Q, Hu L, Chen F, Hu Z, Heist RS, Su L, Amos CI, Shen H, Christiani DC. Polymorphisms in MicroRNAs are associated with survival in non-small cell lung cancer. Cancer Epidemiol Biomarkers Prev. 2014; 23:2503-2511.

10. Mocellin S, Verdi D, Pooley KA, Nitti D. Genetic variation and gastric cancer risk: a field synopsis and meta-analysis. Gut. 2015; 64:1209-1219.

11. Sakamoto H, Yoshimura K, Saeki N, Katai H, Shimoda T, Matsuno Y, Saito D, Sugimura H, Tanioka F, Kato S, Matsukura N, Matsuda N, Nakamura T, Hyodo I, Nishina T, Yasui $\mathrm{W}$, et al. Genetic variation in PSCA is associated with susceptibility to diffuse-type gastric cancer. Nat Genet. 2008; 40:730-740.

12. Abnet CC, Freedman ND, Hu N, Wang Z, Yu K, Shu XO, Yuan JM, Zheng W, Dawsey SM, Dong LM, Lee MP, Ding T, Qiao YL, Gao YT, Koh WP, Xiang YB, et al. A shared susceptibility locus in PLCE1 at 10q23 for gastric adenocarcinoma and esophageal squamous cell carcinoma. Nat Genet. 2010; 42:764-767.

13. Wang LD, Zhou FY, Li XM, Sun LD, Song X, Jin Y, Li JM, Kong GQ, Qi H, Cui J, Zhang LQ, Yang JZ, Li JL, Li XC, Ren JL, Liu ZC, et al. Genome-wide association study of esophageal squamous cell carcinoma in Chinese subjects identifies susceptibility loci at PLCE1 and C20orf54. Nat Genet. 2010; 42:759-763.

14. Shi Y, Hu Z, Wu C, Dai J, Li H, Dong J, Wang M, Miao X, Zhou Y, Lu F, Zhang H, Hu L, Jiang Y, Li Z, Chu M, Ma H, et al. A genome-wide association study identifies new susceptibility loci for non-cardia gastric cancer at 3q13.31 and 5p13.1. Nat Genet. 2011; 43:1215-1218.

15. Gleason CE, Lu D, Witters LA, Newgard CB, Birnbaum MJ. The role of AMPK and mTOR in nutrient sensing in pancreatic beta-cells. J Biol Chem. 2007; 282:1034110351.

16. Dong Y, Chen J, Chen Z, Tian C, Lu H, Ruan J, Yang W. Evaluating the Association of Eight Polymorphisms with Cancer Susceptibility in a Han Chinese Population. PloS one. 2015; 10:e0132797.

17. He J, Qiu LX, Wang MY, Hua RX, Zhang RX, Yu HP, Wang YN, Sun MH, Zhou XY, Yang YJ, Wang JC, Jin L, Wei QY, Li J. Polymorphisms in the XPG gene and risk of gastric cancer in Chinese populations. Hum Genet. 2012; 131:1235-1244.

18. He J, Xu Y, Qiu LX, Li J, Zhou XY, Sun MH, Wang JC, Yang YJ, Jin L, Wei QY, Wang Y. Polymorphisms in ERCC1 and XPF genes and risk of gastric cancer in an eastern Chinese population. PloS one. 2012; 7:e49308.

19. He J, Wang MY, Qiu LX, Zhu ML, Shi TY, Zhou XY, Sun MH, Yang YJ, Wang JC, Jin L, Wang YN, Li J, Yu HP, 
Wei QY. Genetic variations of mTORC1 genes and risk of gastric cancer in an Eastern Chinese population. Mol Carcinog. 2013; 52:E70-79.

20. Wang X, Lu M, Qian J, Yang Y, Li S, Lu D, Yu S, Meng W, Ye W, Jin L. Rationales, design and recruitment of the Taizhou Longitudinal Study. BMC Public Health. 2009; 9:223.

21. Qiu LX, Zhang J, Zhu XD, Zheng CL, Sun S, Wang ZH, Zhao XM, Wang JL, Wang LP, Yu H, Xue K, Hu XC. The p21 Ser31Arg polymorphism and breast cancer risk: a meta-analysis involving 51,236 subjects. Breast Cancer Res Treat. 2010; 124:475-479.

22. Zhu J, Wang M, Zhu M, He J, Wang JC, Jin L, Wang XF, Xiang JQ, Wei Q. Associations of PI3KR1 and mTOR polymorphisms with esophageal squamous cell carcinoma risk and gene-environment interactions in Eastern Chinese populations. Sci Rep. 2015; 5:8250.

23. Zintzaras E, Ioannidis JP. Heterogeneity testing in metaanalysis of genome searches. Genet Epidemiol. 2005; 28:123-137.

24. Kim YD, Yim DH, Eom SY, Moon SI, Yun HY, Song YJ, Youn SJ, Hyun T, Park JS, Kim BS, Lee JY, Won HK, Kim H. Risk of gastric cancer is associated with PRKAA1 gene polymorphisms in Koreans. World JGastroenterol. 2014; 20:8592-8598.

25. Li M, Huang L, Qiu H, Fu Q, Li W, Yu Q, Sun L, Zhang L, $\mathrm{Hu} \mathrm{G}, \mathrm{Hu} J$, Yuan X. Helicobacter pylori infection synergizes with three inflammation-related genetic variants in the GWASs to increase risk of gastric cancer in a Chinese population. PloS one. 2013; 8:e74976.

26. Song HR, Kim HN, Kweon SS, Choi JS, Shim HJ, Cho SH, Chung IJ, Park YK, Kim SH, Choi YD, Joo KW, Shin MH. Genetic variations in the PRKAA1 and ZBTB20 genes and gastric cancer susceptibility in a Korean population. Mol Carcinog. 2013; 52:E155-160.

27. Hou L, Li Y, Song H, Zhang Z, Sun Y, Zhang X, Wu K. Protective Macroautophagy Is Involved in Vitamin E Succinate Effects on Human Gastric Carcinoma Cell Line SGC-7901 by Inhibiting mTOR Axis Phosphorylation. PloS one. 2015 ; 10:e 0132829 .
28. Li S, Zhou T, Li C, Dai Z, Che D, Yao Y, Li L, Ma J, Yang X, Gao G. High metastaticgastric and breast cancer cells consume oleic acid in an AMPK dependent manner. PloS one. 2014; 9:e97330.

29. Kim YH, Liang H, Liu X, Lee JS, Cho JY, Cheong JH, Kim H, Li M, Downey TJ, Dyer MD, Sun Y, Sun J, Beasley EM, Chung HC, Noh SH, Weinstein JN, et al. AMPKalpha modulation in cancer progression: multilayer integrative analysis of the whole transcriptome in Asian gastric cancer. Cancer Res. 2012; 72:2512-2521.

30. Obba S, Hizir Z, Boyer L, Selimoglu-Buet D, Pfeifer A, Michel G, Hamouda MA, Goncalves D, Cerezo M, Marchetti S, Rocchi S, Droin N, Cluzeau T, Robert G, Luciano F, Robaye B, et al. The PRKAA1/AMPKalpha1 pathway triggers autophagy during CSF1-induced human monocyte differentiation and is a potential target in CMML. Autophagy. 2015; 11:1114-1129.

31. Lee SJ, Kang BW, Chae YS, Kim HJ, Park SY, Park JS, Choi GS, Jeon HS, Lee WK, Kim JG. Genetic variations in STK11, PRKAA1, and TSC1 associated with prognosis for patients with colorectal cancer. Ann Surg Oncol. 2014; 21:S634-639.

32. Bhandaru M, Martinka M, Li G, Rotte A. Loss of AMPKalphal expression is associated with poor survival in melanoma patients. J Invest Dermatol. 2014; 134: 1763-1766.

33. Hwang JY, Kim DH, Ji YI, Jin Go M, Heo L, Jin Kim Y, Sung Sohn T, Hyung Noh J, Kim S, Lee YS, Kim SY, Kim YW, Won Ryu K, Choi IJ, Lee J, Kim BJ, et al. Recapitulation of previous genome-wide association studies with two distinct pathophysiological entities of gastric cancer in the Korean population. J Hum Genet. 2013; 58:233-235. 